\title{
Expression of MicroRNA-301a and its Functional Roles in Malignant Melanoma
}

\author{
Lei Cuia,b Yuejun Lia Xiaoxing Lva Jinqing Lia Xiaolin Wanga ${ }^{a}$ Zhanjun Lei ${ }^{a}$ \\ Xueyong Lia
}

aDepartment of Plastic Surgery, Tangdu Hospital, Fourth Military Medical University, Xi'an, ${ }^{b}$ Department of Burn and Plastic Surgery, Jinling Hospital, School of Medicine, Nanjing University, Nanjing, PR China

\author{
Key Words \\ MicroRNA-301a • PTEN • Melanoma • Growth • Invasion • Migration • Chemoresistance
}

\begin{abstract}
Background/Aims: Although microRNA-301a has been reported to function as an oncogene in many human cancers, the roles of miR-301a in malignant melanoma (MM) is unclear. The present study aims to investigate the functional roles of miR-301a in MM and its possible molecular mechanisms. Methods: Quantitative real-time PCR (qRT-PCR) assay was performed to detect the expression of miR-301a in MM tissues, and analyze its correlation with metastasis and prognosis of MM patients. In vitro, miR-301a was ectopically expressed using overexpression and knock-down strategies, and the effects of miR-301a expression on growth, apoptosis, migration, invasion and chemosensitivity of MM cells were further investigated. Furthermore, the potential and functional target gene was identified by luciferase reporter, qRT-PCR, Western blot assays. Results: We showed that the expression of miR-301a was significantly upregulated in MM tissues, and upregulation of miR-301a correlated with metastasis and poor prognosis of MM patients. Transfection of miR-301a/inhibitor significantly inhibited growth, colony formation, migration, invasion and enhanced apoptosis and chemosensitivity in $\mathrm{MM}$ cells, while transfection of miR-301a/mimic could induce the inverse effects on phenotypes of MM cells. Luciferase reporter, qRT-PCR and Western blot assays showed that phosphatase and tensin homolog (PTEN) was a direct and functional target of miR-301a. It was also observed that the Akt and FAK signaling pathways were involved in miR-301/PTEN-promoting MM progression. Conclusion: Taken together, our study suggests that miR-301a may be used as a potential therapeutic target in the treatment of human MM.
\end{abstract}

\section{Introduction}

Malignant melanoma (MM) is a highly aggressive cancer with a high metastatic potential and rapidly increasing incidence around the world [1]. Currently, a wide surgical resection is the main curative treatment option for most MM cases with localized disease. However, for patients with advanced MM, the curative effects of conventional treatments including 
systemic chemotherapy, molecular-targeted therapy, radiotherapy and immunotherapy are still quite limited [2]. Therefore, it is urgent to develop novel therapeutic strategy for metastatic MM. The carcinogenesis of MM is a multi-step and complicated process, which involves activation of oncogenes and inactivation of tumor suppressor genes [3]. Thus, a better understanding of the genetic and molecular basis of MM progression will contribute to exploit novel therapeutic targets for MM patients.

It is well known that microRNAs (miRNAs) are a family of noncoding RNAs 22 nucleotides in length, which suppress gene expression by binding to the 3' untranslated regions (3'-UTRs) of target mRNAs [4]. The aberrant expression of miRNAs has been reported to play multiple roles in various cellular processes, including differentiation, metablism, apoptosis, development and tumorigenesis [5-9]. Numerous studies have shown that dysregulation of miRNAs plays a critical role in MM development. The correlations of miRNAs with prognosis of MM patients are reported, such as miR-10b, miR-106b, miR-206, etc [10-12]. On the one hand, some miRNAs are found to function as tumor suppressors in MM cells. For example, Rang et al. showed that miR-542-3p suppresses invasion and metastasis of melanoma cells by targeting the proto-oncogene serine/threonine protein kinase (PIM1) [13]. Sun et al. also showed that miR-144 inhibits proliferation and invasion of uveal melanoma cells by targeting c-Met [14]. On the other hand, other miRNAs are reported to function as oncogenes in MM cells. For example, Ren et al. showed that miR-135 promotes cell proliferation of MM cells by regulation of FOXO1 expression [15]. Sun and his colleagues reported that miR-454 functions as an oncogene in uveal melanoma by targeting PTEN [16]. It has been reported that miR-301a functions as a candidate oncogene in multiple human cancers, such as hepatocelluar cancer, pancreatic cancer, and Ewing's sarcoma [17-19]. By comparative microarray analysis of microRNA expression profiles in primary cutaneous malignant melanoma (CMM), CMM metastases, and benign melanocytic nevi, Sand and his colleagues identified 19 dysregulated miRNA candidates in CMM samples and among those miRNAs, miR-301a was found to be significantly upregulated [20]. However, the roles of miR-301a in MM progression are unclear and remain to be further explored.

In the present study, we detected the expression of miR-301a in MM tissues. Then, the effects of miR-301a on growth, migration, invasion and chemosensitivity of MM cells were determined. Furthermore, phosphatase and tensin homolog (PTEN) was identified as a direct and functional target of miR-301a, and the downstream signaling pathways were also determined. Thus, targeting miR-301a may be a promosing strategy for MM therapy.

\section{Materials and Methods}

\section{Cell culture}

The human MM cell lines (SK-MEL-1 and A-375) were purchased from the Shanghai Institute of Cell Biology, Chinese Academy of Sciences (Shanghai, China). All cells were grown in Dulbecco's Modified Eagle's medium (DMEM; Gibco, USA) supplemented with $10 \%$ fetal bovine serum at $37^{\circ} \mathrm{C}$ in $5 \% \mathrm{CO}_{2}$ atmosphere.

Tissue samples

The 18 cases of benign melanocytic naevi (BMN) tissues and 46 cases of MM tissues used in this study were obtained from patients who underwent radical operations between January 2008 and Dec 2009. Approval from the institutional ethics committee and the patients' written informed consent were obtained before surgery. This study was approved by the Ethics Committee of the hospital. The written informed consents had been signed by all patients in advance. None patients had received any radiotherapy or chemotherapy prior to surgery. All tissues were snap frozen in liquid nitrogen, followed by storage at $-80^{\circ} \mathrm{C}$ until use.

Transfection of oligonucleotides or plasmids

The miR-301a (miR-301a-3p) mimics (miR-301a/mimic) or inhibitor (miR-301a/inhibitor) and their negative control oligonucleotides (control/mimic or control/inhibitor) were obtained from GenePharma 


\section{Cellular Physiology Cell Physiol Biochem 2016;40:230-244 \begin{tabular}{l|l|l} 
and BOI: 10.1159/000452540 & $\begin{array}{l}\text { C } 2016 \text { The Author(s). Published by S. Karger AG, Basel } \\
\text { www.karger.com/cpb }\end{array}$
\end{tabular}}

Cui et al.: MiR-301a: a Potential Therapeutic Target for Malignant Melanoma

Co., Ltd (Shanghai, China). The plasmid vector (pMD/PTEN) expressing open reading frame of PTEN was purchased from Sino Biological Inc. (Beijing, China). The recombinant vectors were confirmed by the digestion analysis of restriction endonuclease and DNA sequencing. Transient or stable transfections were performed using Lipofectamine ${ }^{\mathrm{TM}} 2000$ (Invitrogen, USA) according to the manufacturer's instructions.

Quantitative real-time PCR ( $q R T-P C R)$ assay

Total RNA from cells and tissues and was extracted with the TRIzol reagent (Invitrogen, USA) the PCR analyses were performed according to the MIQUE (minimum information for publication of quantitative real-time PCR experiments) guidelines [21]. The expression level of miR-301a were quantified by using miRNA-specific TaqMan MiRNA Assay Kit (Applied Biosystems) and expression of RNU6B (Qiagen, USA) was used as endogenous control [22]. For determining the expression of PTEN mRNA, total RNA was reverse transcribed to cDNA and qRT-PCR was performed as described previously and expression of GAPDH was used as endogenous control. The primers of PTEN and GAPDH mRNA for qRT-PCR detection were as follows: PTEN, forward: 5'-TAGAGGAGCCGTCAAATC-3'; reverse, 5'-ATCAGAGTCAGTGGTGTC-3'; GAPDH, forward: 5'-GGAAGGTGAAGGTCGGAGTCA-3'; reverse: 5'-GTCATTGATGGCAACAATATCCACT-3'. Each experiment was performed in triplicate.

\section{Western blot assay}

The cells were washed with prechilled PBS and solubilized in 1\% Nonidet P-40 lysis buffer. Homogenates were clarified by centrifugation at $20,000 \times \mathrm{g}$ for $15 \mathrm{~min}$ at $4^{\circ} \mathrm{C}$ and the protein concentration was measured by bicinchoninic acid protein assay kit (Pierce Biotechnology). Proteins were separated by sodium dodecyl sulfate-polyacrylamide gel electrophoresis (SDS-PAGE). All the specific antibodies against phosphorylated AKT (p-Akt) (Thr308), total AKT, phosphorylated FAK (Tyr397) and (Tyr577), total FAK, Bcl-2, Bax, MMP-2 and 9 proteins were purchased from Santa Cruz Biotechnology (CA, USA) or Cell Signaling Technology (Beverly, MA, USA). The membranes were then incubated with a horseradish peroxidaseconjugated secondary antibody (Sigma, USA). Probing and detection of target proteins was performed by an enhanced chemiluminescence (ECL) assay.

\section{MTT assay}

The cell viability was examined by MMT assay as previously described [23]. In brief, the transfected cells were seeded into 96 well plates at $5.0 \times 10^{3}$ cells/well, and $20 \mu \mathrm{MTT}(5 \mathrm{mg} / \mathrm{mL})$ was added and incubated for $4 \mathrm{~h}$. Then, $150 \mu \mathrm{L}$ of DMSO was added to each well to dissolve the precipitate. Optical density (OD) was measured at the wavelength of $490 \mathrm{~nm}$. Each test was repeated in six wells and performed in triplicate.

\section{Colony formation assay}

The cells were placed in a fresh six-well plate (600 cells/well) and maintained in DMEM containing $10 \%$ FBS. After 14 days, cells were fixed with methanol and stained with $0.1 \%$ crystal violet. Visible colonies were manually counted and each experiment was performed in triplicate.

\section{Flow cytometric analysis of apoptosis}

The cells were collected and the apoptosis was detected using an Annexin V-fluorescein isothiocyanate (FITC) apoptosis detection kit (BioVision, Mountain View, CA, USA) according to the manufacturer's instructions. Each experiment was performed in triplicate.

Wound scratch healing or transwell invasion assay

The cell migration or invasion was measured using wound scratch healing or transwell invasion assay as previously described [24]. Each experiment was performed in triplicate.

\section{In vitro chemosensitivity assay}

The single-cell suspensions were prepared and dispersed in 96-well plates. After incubation for 72 $\mathrm{h}$ with the cisplatin (CDDP) or adriamycin (ADR) compounds (Sigma, MO, USA), the $0.5 \mathrm{mg} / \mathrm{mL}$ of MTT solution (Sigma, USA) was added. Following incubation for $4 \mathrm{~h}, 100 \mu \mathrm{L}$ of extraction buffer were added to each well. After an overnight incubation, absorbance at $490 \mathrm{~nm}$ was measured using a microplate reader. Each experiment was performed in triplicate. 


\section{Cellular Physiology Cell Physiol Biochem 2016;40:230-244

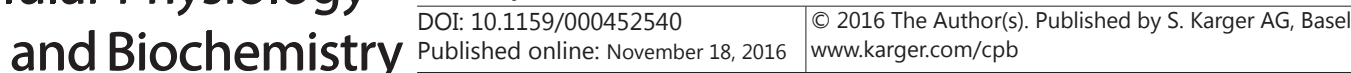

Cui et al.: MiR-301a: a Potential Therapeutic Target for Malignant Melanoma

\section{Luciferase reporter assay}

The MM cell line (A-375) was grown in a 48-well plate were co-transfected with miR-301a/mimic (or control/mimic) or miR-301a/inhibitor (control/inhibitor), and pLUC firefly luciferase vectors containing wild-type or mutant PTEN 3'-UTR sequence (PTEN/3'-UTR-wt or PTEN/3'-UTR-mut) using Lipofectamine ${ }^{\text {TM }}$ 2000 (Invitrogen, USA) as previously described [25, 26]. Co-transfected with $48 \mathrm{~h}$ post-transfection, the cells were lysed for measurement of luciferase activities using the Dual-Luciferase Assay kit (Promega, Madison, WI, USA) according to the manufacturer's instructions. Relative luciferase activity was calculated by normalizing the ratio of Firefly/Renilla luciferase to that of negative control-transfected cells.

\section{Statistical analysis}

All statistical analyses were performed using statistical software (SPSS17.0 for Windows; SPSS, Inc., Chicago, IL). Spearman correlation test was used for analyses of primary tumors. Survival probabilities were determined using Kaplan-Meier analysis and the significance of difference was analyzed by a log-rank test. Significance was accepted at $P<0.05$. Data were expressed as mean \pm SEM from at least three independent experiments.

\section{Results}

Upregulation of miR-301 a correlates with MM progression

First, qRT-PCR was performed to detect the expression of miR-301a in 18 cases of BMN tissues and 46 cases of MM tissues. As shown in Fig. 1A, the relative expression level of miR$301 \mathrm{a}$ in MM tissues was significantly higher than that in BMN tissues $(P<0.001)$. Also, it was found that the expression of miR-301a in MM tissues with metastasis $(n=24)$ was higher than that in MM tissues without metastasis $(\mathrm{n}=22)(P<0.01$; Fig. 1B). To further analyze the correlation of miR-301a expression with prognosis of MM patients, patients with values less than the median expression level in tumor tissues were assigned to the low-miR-301a

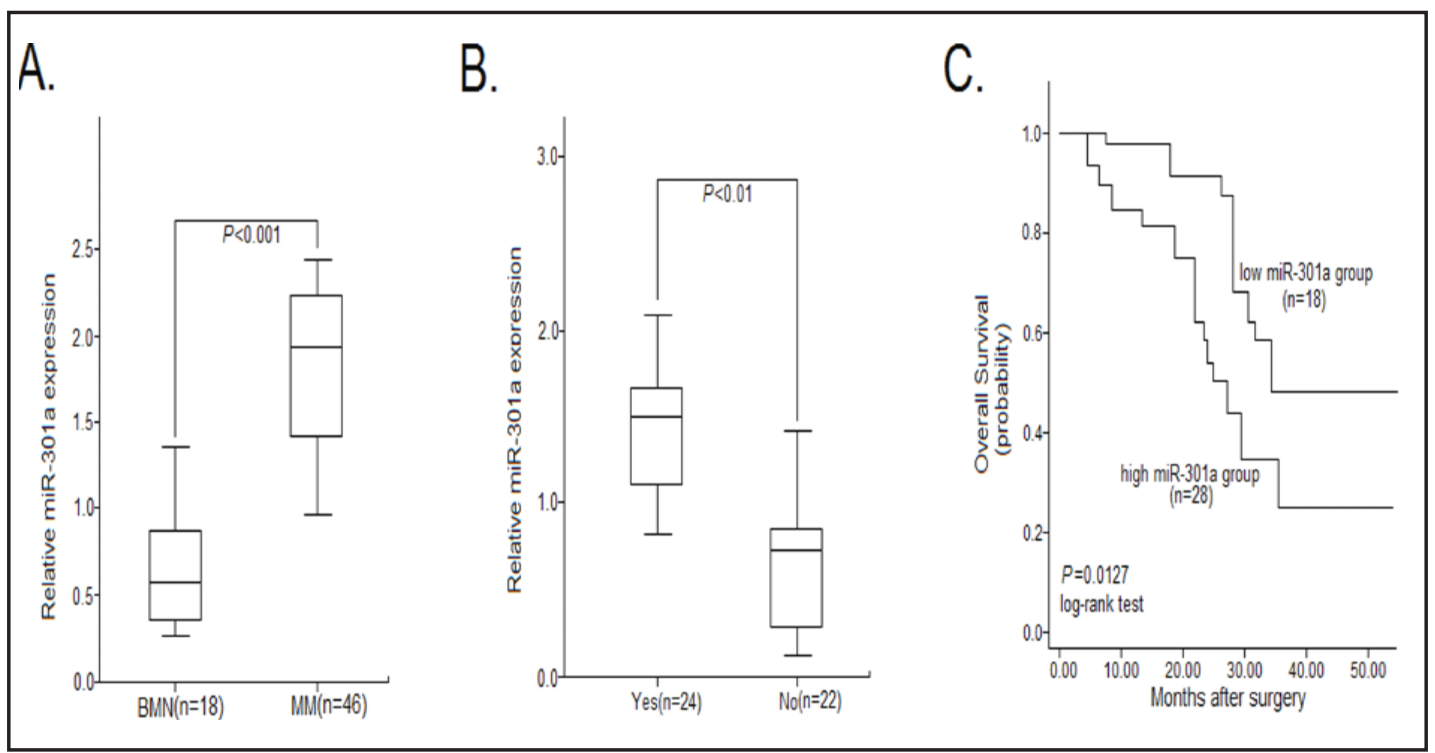

Fig. 1. Expression of miR-301a is upregulated in MM tissue samples. (A) qRT-PCR detection of the expression of miR-301a in BMN tissues $(n=18)$ is significantly lower than that in MM tissues $(n=46)$. (B) The expression of miR-301a in MM tissues with metastasis $(n=24)$ is higher than that in MM tissues without metastasis $(\mathrm{n}=22)$. C) MM patients with high-miR-301a expression $(\mathrm{n}=28)$ show poorer prognosis than those with low-miR-301a expression $(\mathrm{n}=18)$. U6 RNA was used as the internal control. Error bars indicate the standard deviation. Results represent the average of three independent experiments (mean $\pm \mathrm{SD}$ ). ${ }^{*} P<$ 0.05 and $^{* *} P<0.01$. 
A.

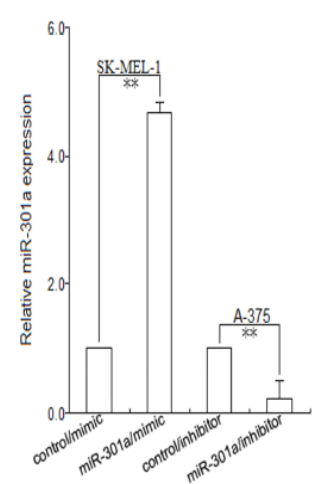

C.
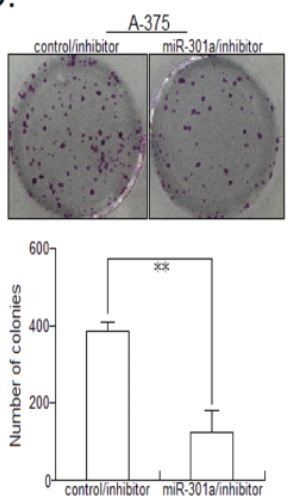

B.
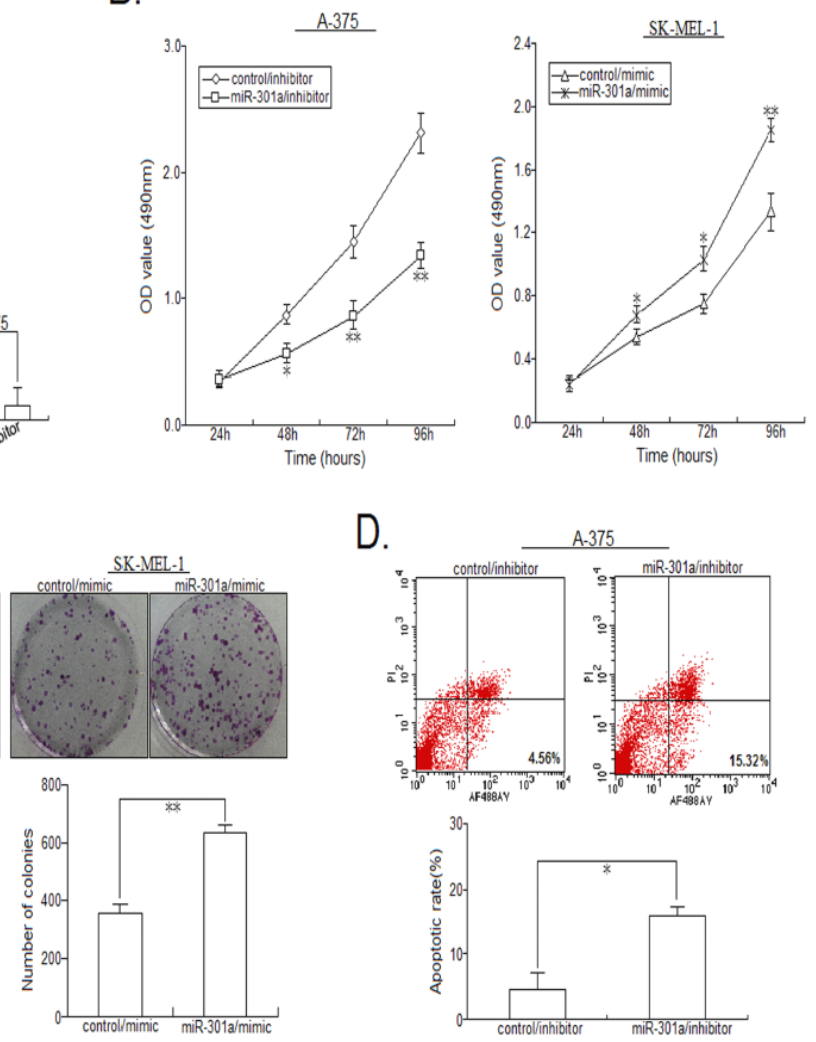

D.
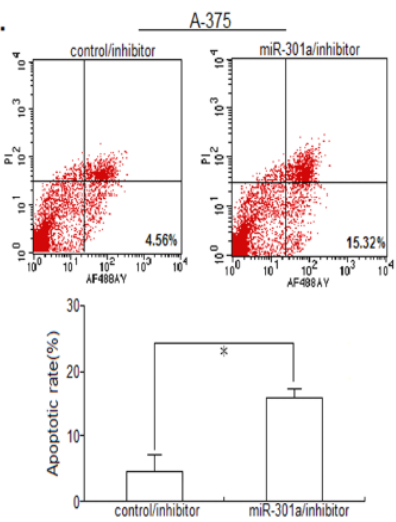

Fig. 2. Effect of miR-301a expression on growth, colony formation and apoptosis in MM cells. (A) qRT-PCR detection of miR-301a expression in MM cell line (SK-EML-1) transfected with miR-301a/mimic (control/ mimic) or MM cell line (A-375) transfected with miR-301a/inhibitor (control/inhibitor), respectively. U6 RNA was used as the internal control. (B) MTT analysis of growth in the transfected MM cells. The OD values are measured at the different time point. (C) Representative results of colony formation of the transfected MM cells. (D) Flow cytometric detection of apoptosis in A-375 cells transfected with miR-301a/inhibitor or control/inhibitor. Error bars indicate the standard deviation. Results represent the average of three independent experiments (mean $\pm \mathrm{SD}$ ). $* P<0.05$ and ${ }^{* *} P<0.01$.

group ( $\mathrm{n}=18)$, whereas those with values more than the median expression level were assigned to the high-miR-301a group $(\mathrm{n}=28)$. As shown in Fig. $1 \mathrm{C}, \mathrm{MM}$ patients with highmiR-301a expression had poorer overall survival than those with low-miR-301a expression $(P=0.0127)$. These results suggest that upregulation of miR-301a may play a role in MM progression.

Effect of miR-301a expression on proliferation, colony formation and apoptosis in MM cells

To explore the functional roles of miR-301a in MM cells, SK-EML-1 or A-375 cell line was transfected with miR-301a/mimic (or control/mimic) or miR-301a/inhibitor (or control/ inhibitor), respectively. Then, qRT-PCR confirmed upregulation of miR-301a in SK-EML-1 cells transfected with miR-301a/mimic and downregulation of miR-301a in A-375 cells transfected with miR-301a/inhibitor, in comparison with control cells, respectively $(P<$ 0.01; Fig. 2A). First, the effect of miR-301a expression on growth of MM cells was determined by MTT and colony formation assays. Downregulation of miR-301a significantly reduced growth and colony formation in A-375 cells, while re-expression of miR-301a led to the increased capacities of growth and colony formation in SK-EML-1 cells (Fig. 2B-C). Then, the effect of miR-301a downregulation on apoptosis of MM cells was also determined. It was found that transfection of miR-301a/inhibitor increased apoptosis of SK-EML-1 cells by 
A.
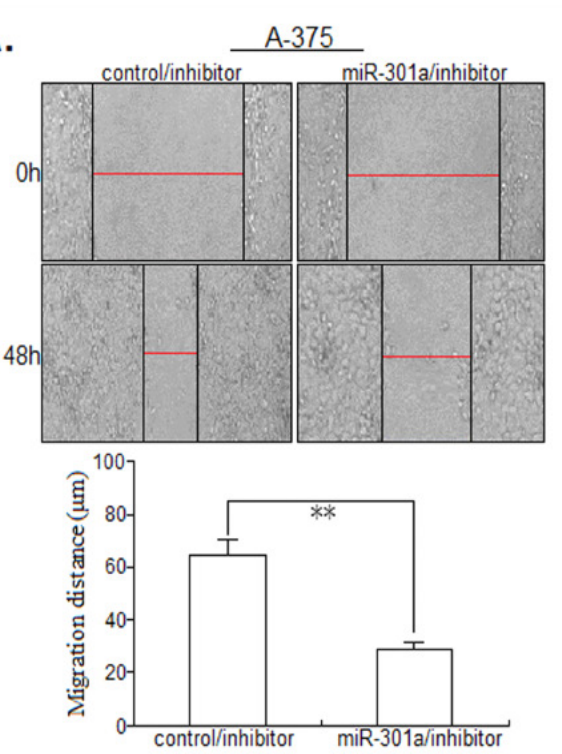

C.
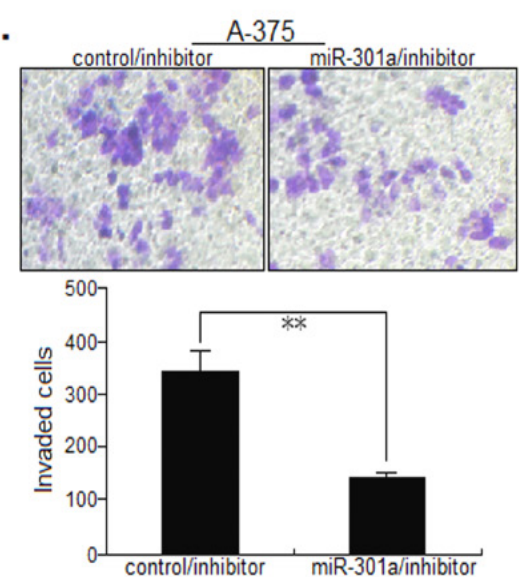

B.
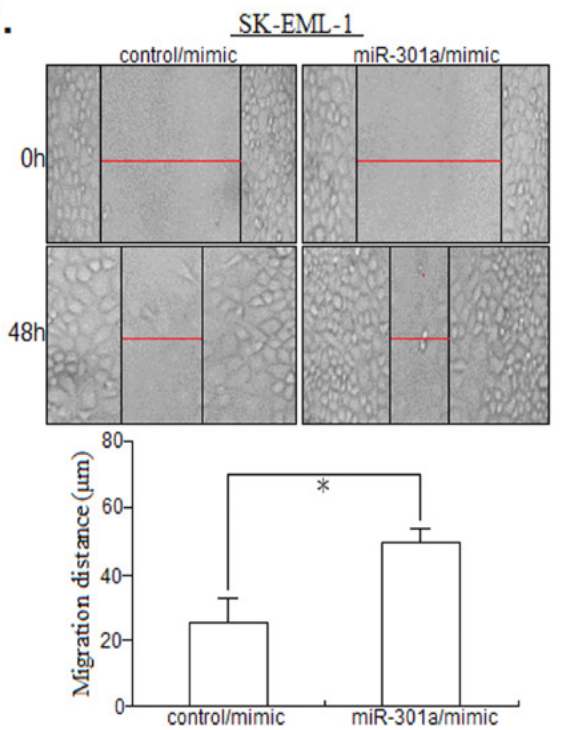

D.
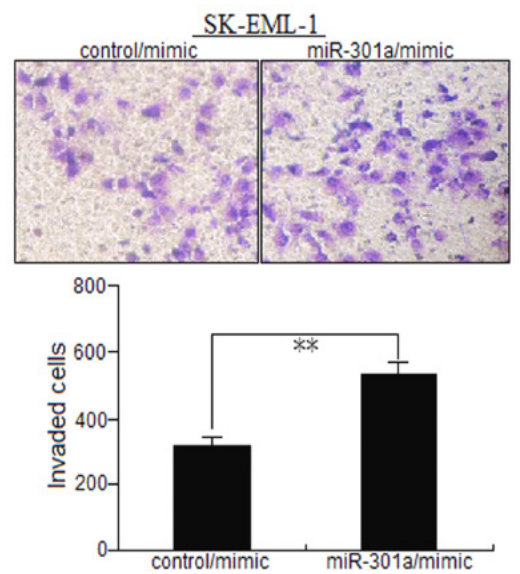

Fig. 3. Effect of miR-301a expression on the capacities of migration and invasion in MM cells. (A) Scratch wound healing assay of migration in A-375 cells transfected with miR-301a/inhibitor (control/inhibitor) or SK-EML-1 transfected with miR-301a/mimic (control/mimic), respectively. (B) Matrigel transwell invasion assay of invasion in A-305 cells transfected with miR-301a/inhibitor (control/inhibitor) or SK-EML-1 transfected with miR-301a/mimic (control/mimic), respectively. Error bars indicate the standard deviation. Results represent the average of three independent experiments (mean \pm SD). ${ }^{*} P<0.05$ and ${ }^{* *} P<0.01$.

about $10.76 \%(P<0.05$; Fig. 2D). These data indicate that miR-301a may be a promoter of growth in MM progression.

Effect of miR-301a expression on the capacities of migration and invasion in MM cells

To further investigate the effect of miR-301a expression on the capacities of migration and invasion in MM cells, wound healing and Transwell matrigel invasion assays were performed. As shown in Fig. 3A-B, downregulation of miR-301a significantly reduced the capacities of migration and invasion in A-375 cells $(P<0.01)$, while re-expression of miR$301 \mathrm{a}$ led to the increased capacities of migration and invasion in SK-EML-1 cells $(P<0.05)$. Therefore, miR-301 promotes migration and invasion in MM cells.

Effect of miR-301a expression on the chemosensitivity of MM cells

Next, we investigated whether miR-301a protects MM cells against cytotoxicity mediated by chemotherapeutic agents (CDDP and ADR). The miR-301a/inhibitor (or control/inhibitor)- 


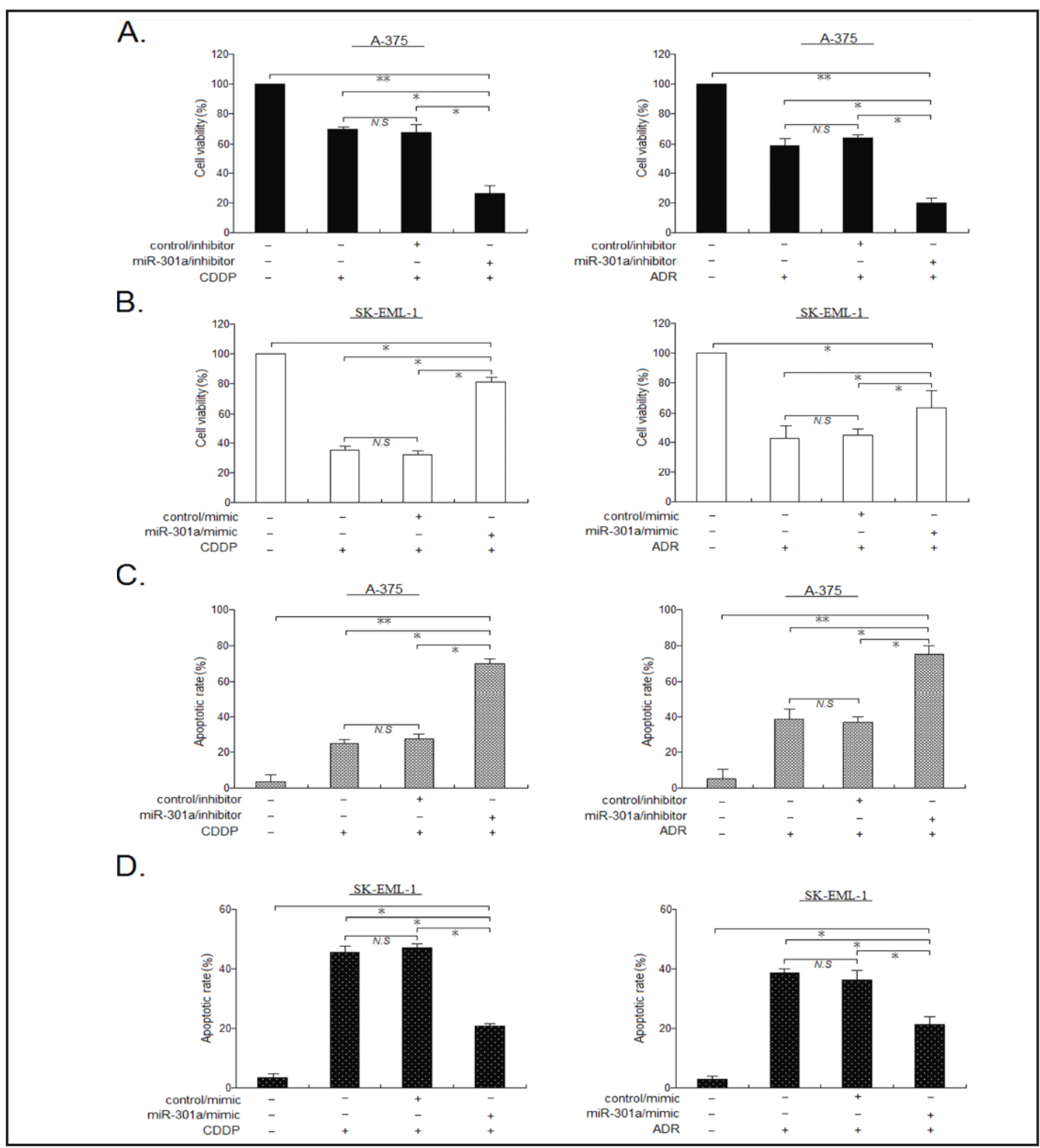

Fig. 4. Effect of miR-301a expression on the chemosensitivity of MM cells to CDDP or ADR. (A) MMT analysis of growth in miR-301a/inhibitor or control/inhibitor-transfected A-375 cells treated with CDDP (8 $\mu \mathrm{g} / \mathrm{ml})$ or ADR $(1 \mu \mathrm{M})$, respectively. (B) MMT analysis of growth in miR-301a/mimic or control/mimic-transfected SK-EML-1 cells treated with CDDP $(8 \mu \mathrm{g} / \mathrm{ml})$ or ADR $(1 \mu \mathrm{M})$, respectively. (C) Flow cytometric detection of apoptosis in miR-301a/inhibitor or control/inhibitor-transfected A-375 cells treated with CDDP $(8 \mu \mathrm{g} / \mathrm{ml})$ or ADR $(1 \mu \mathrm{M})$, respectively. (D) Flow cytometric detection of apoptosis in miR-301a/mimic or control/ mimic-transfected SK-EML-1 cells treated with CDDP $(8 \mu \mathrm{g} / \mathrm{ml})$ or ADR $(1 \mu \mathrm{M})$, respectively. Error bars indicate the standard deviation. Results represent the average of three independent experiments (mean \pm SD). * $P<0.05,{ }^{* *} P<0.01$ and N.S, $P>0.05$.

transfected A-375 cells or miR-301a/mimic (or control/mimic)-transfected SK-EML-1 cells were treated with CDDP $(8 \mu \mathrm{g} / \mathrm{ml})$ or ADR $(1 \mu \mathrm{M})$ for $48 \mathrm{~h}$, and then the cells were collected for further assays. First, we determined the changes of cell viability by MTT assay. As shown in Fig. 4A-B, transfection of miR-301a/inhibitor significantly enhanced the cytotoxicity of CDDP or ADR in A-375 cells $(P<0.05)$, while transfection of miR-301a/mimic moderately 
A.

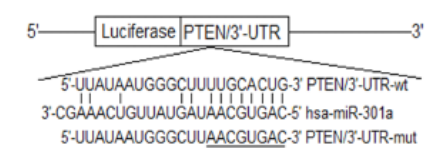

C.

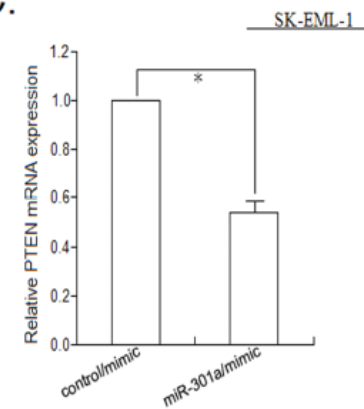

E.

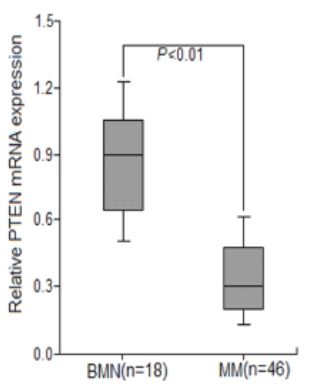

B.
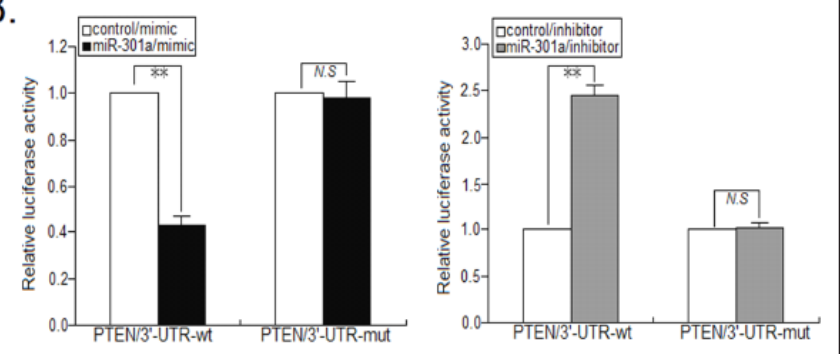

D.

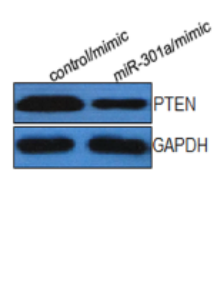

F.

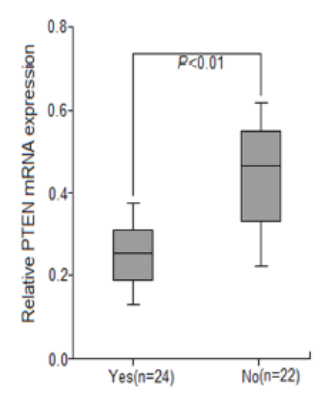

G.

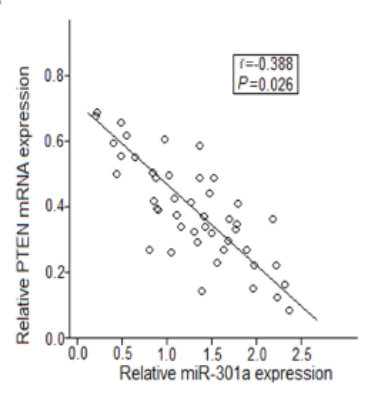

Fig. 5. Identification of PTEN as a direct target of miR-301a in MM cells. (A) Putative miR-301a complementary site in the 3'-UTR of PTEN mRNA. A mutated 3'-UTR of PTEN mRNA for the miR-301a complementary site was generated. (B) Luciferase reporter assay was performed by co-transfection of A-375 cells with luciferase reporter containing the wild-type 3'-UTR of PTEN (PTEN/3'-UTR-wt) or mutant 3'-UTR of PTEN (PTEN/3'-UTR-mut) with miR-301a/mimic (or control/mimic) or miR-301a/inhibitor (or control/ inhibitor), respectively. Luciferase activity was determined $48 \mathrm{~h}$ after co-transfection. (C) qRT-PCR and Western blot detection of PTEN mRNA and protein expression in SK-EML-1 cells transfected with miR-301a/ mimic or control/mimic, respectively. (D) qRT-PCR and Western blot detection of PTEN mRNA and protein expression in A-375 cells transfected with miR-301a/inhibitor or control/inhibitor, respectively. GAPDH was used as the internal control. (E) The relative level of PTEN mRNA expression in BMN tissues $(\mathrm{n}=18)$ was significantly higher that that in MM tissues $(\mathrm{n}=46)(P<0.01)$. (F) The relative level of PTEN mRNA expression in MM tissues with metastasis $(\mathrm{n}=24)$ was significantly higher that that in MM tissues with no metastasis $(\mathrm{n}=22)(P<0.01)$. $(\mathrm{G})$ The Spearman correlation indicated an inverse relation between miR301a and PTEN mRNA in the MM tissues ( $\mathrm{n}=46 ; \mathrm{r}=-0.338 ; P=0.026)$. Error bars indicate the standard deviation. Results represent the average of three independent experiments (mean \pm SD). ${ }^{*} P<0.05$, ${ }^{* *} P<$ 0.01 and N.S, $P>0.05$.

reduced the cytotoxicity of CDDP or ADR in SK-EML-1 cells $(P<0.05)$. Then, we analyzed the changes of apoptosis by flow cytometry assay. As shown in Fig. 4C-D, downregulation of miR-301a increased the CDDP or ADR-induced apoptosis in A-375 cells $(P<0.01)$, while upregulation of miR-301a decreased the CDDP or ADR-induced apoptosis in SK-EML-1 cells $(P<0.05)$. These results suggest that miR-301a decreases the chemosensitivity of MM cells by reducing chemotherapy-induced apoptosis. 
A.

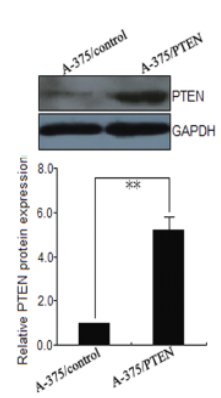

D.
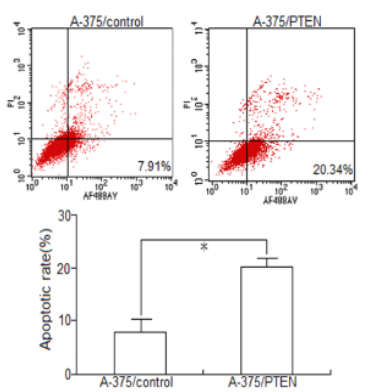

G.

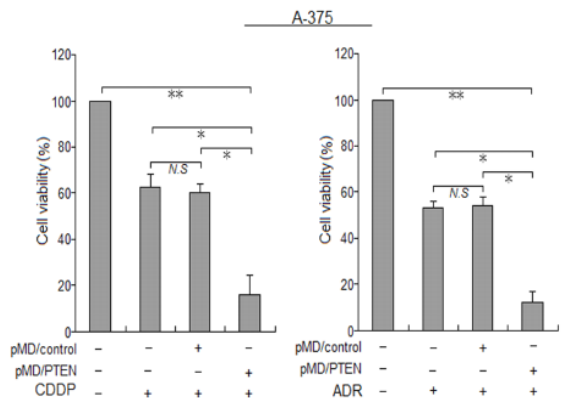

B.

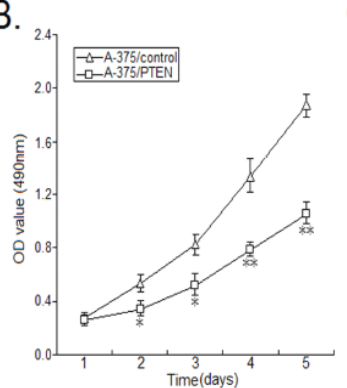

E.

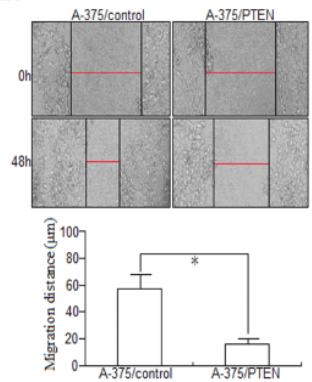

C.
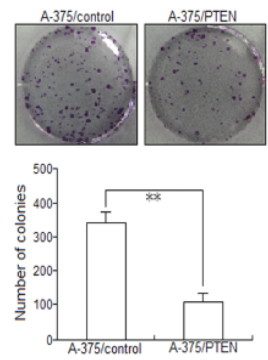

F.

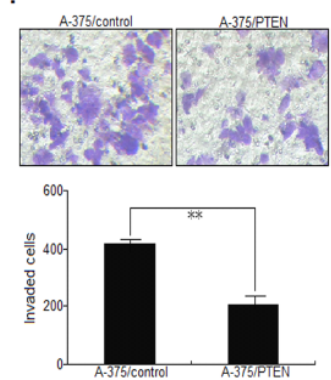

$\mathrm{H}$.

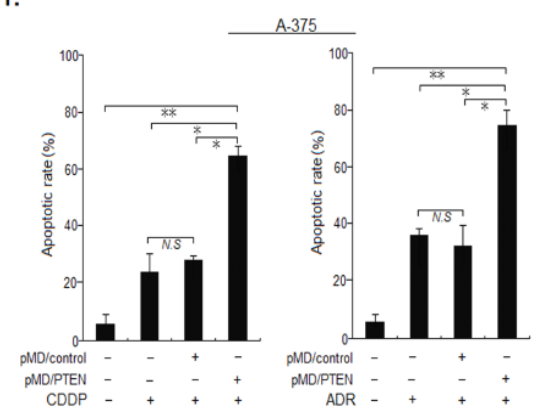

Fig. 6. Effect of PTEN upregulation on malignant phenotypes of MM cells. (A) Western blot detection of PTEN protein expression in the stably transfected A-375/PTEN or A-375/control cells, respectively. GAPDH was used as the internal control. (B) MTT analysis of growth in A-375/PTEN or A-375/control cells, respectively. (C) Representative results of colony formation of A-375/PTEN or A-375/control cells, respectively. (D) Flow cytometric detection of apoptosis A-375/PTEN or A-375/control cells, respectively. (E) Scratch wound healing assay of migration in A-375/PTEN or A-375/control cells, respectively. (F) Matrigel transwell invasion assay of invasion in A-375/PTEN or A-375/control cells, respectively. (G) MMT analysis of growth in A-375/PTEN or A-375/control cells treated with CDDP $(8 \mu \mathrm{g} / \mathrm{ml})$ or ADR $(1 \mu \mathrm{M})$, respectively. (H) Flow cytometric detection of apoptosis in A-375/PTEN or A-375/control cells treated with CDDP (8 $\mu \mathrm{g} /$ $\mathrm{ml})$ or ADR $(1 \mu \mathrm{M})$, respectively. Error bars indicate the standard deviation. Results represent the average of three independent experiments (mean $\pm \mathrm{SD}$ ). ${ }^{*} P<0.05$, ${ }^{* *} P<0.01$ and N.S, $P>0.05$.

\section{Identification of PTEN as a direct and functional target of miR-301a in MM cells}

PTEN is one of the most frequently mutated tumor suppressors in human cancers including melanoma. The targets of miR-301a were predicted through at least three databases (Pictarget, miRBas and TargetScan), and PTEN was selected as a putative target, which was also recently reported in other cancers [20,27]. The 3'-untranslated region (UTR) of PTEN gene stood out because of the presence of one evolutionarily conserved binding site (4483 4503nt) for miR-301a. To confirm whether miR-301a can bind to the 3'-UTR of PTEN mRNA, we performed luciferase reporter assay with a vector containing the putative PTEN 3'-UTR target site downstream of the luciferase reporter gene. Base pairing between miR301a and wild-type (wt) or mutant (mut) target site in the 3'-UTR of PTEN mRNA is shown in Fig. 5A. 48 hours after co-transfection of miR-301a/mimic (or control/mimic) or miR-301a/ 


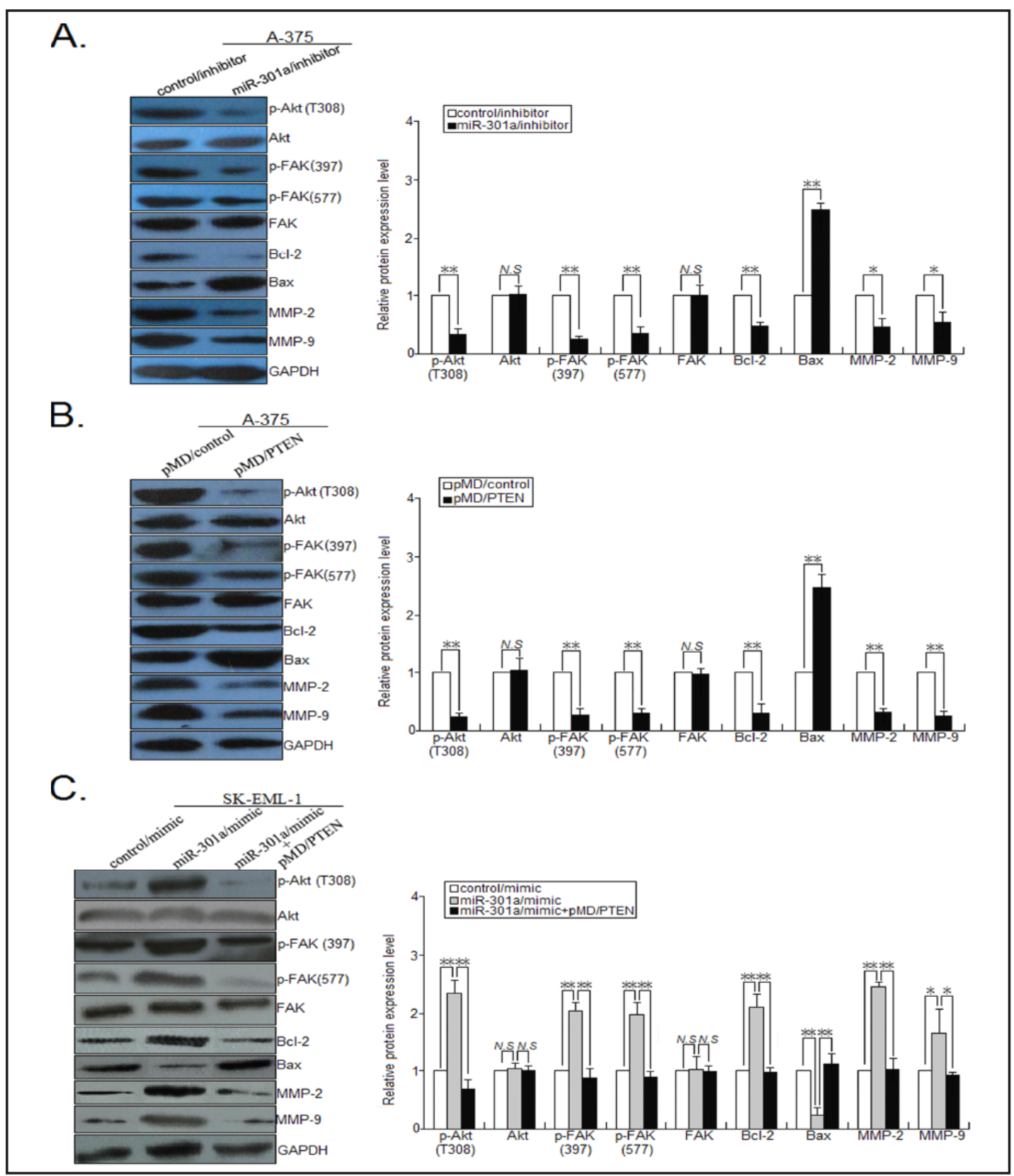

Fig. 7. Effect of miR-301a expression on Akt and FAK signaling pathways. (A) Western blot detection of the expression of p-Akt (T308), total Akt, p-FAK (397), p-FAK(577), total FAK, Bcl-2, Bax, MMP-2, MMP-9 proteins in miR-301a/inhibitor or control/inhibitor-transfected A-375 cells, respectively. (B) Western blot detection of the expression of p-Akt (T308), total Akt, p-FAK (397), p-FAK(577), total FAK, Bcl-2, Bax, MMP2, MMP-9 proteins in A-375/PTEN or A-375/control cells, respectively. (C) Western blot detection of the expression of p-Akt (T308), total Akt, p-FAK (397), p-FAK(577), total FAK, Bcl-2, Bax, MMP-2, MMP-9 proteins in miR-301a/mimic or control/mimic-transfected SK-EML-1 cells or SK-EML-1 cells co-transfected with miR-301a/mimic and pMD/PTEN, respectively. GAPDH was used as the internal control. Error bars indicate the standard deviation. Results represent the average of three independent experiments (mean \pm SD). ${ }^{*} P<0.05,{ }^{* *} P<0.01$ and N.S, $P>0.05$.

inhibitor (control/inhibitor) with pMir-PTEN/3'-UTR-wt or pMir-PTEN/3'-UTR-mut vector, luciferase activity was measured. We showed that the luciferase activity was decreased in A-375 cells co-transfected with miR-301a/mimic and pMir-PTEN/3'-UTR-wt $(P<0.05)$ and 
increased in A-375 cells co-transfected with miR-301a/inhibitor and pMir-PTEN/3'-UTR-wt $(P<0.05)$, compared to controls. However, no changes could be observed in cells which were co-transfected with miR-301a/mimic and pMir-PTEN/3'-UTR-mut $(P>0.05)$ or with miR301a/inhibitor and pMir-PTEN/3'-UTR-mut $(P>0.05)$, compared to controls (Fig. 5B). In addition, we performed Western blot assay to analyze the effect of miR-301a expression on PTEN mRNA and protein expression. Transfection of miR-301a/mimic led to the decreased expression of PTEN mRNA and protein in SK-EML-1 cells, while transfection of miR-301a/ inhibitor led to the increased expression of PTEN mRNA and protein in A-375 cells (Fig. 5CD). Next, we analyzed the expression of PTEN mRNA in MM tissues and its correlation with miR-301a expression. The relative expression of PTEN mRNA in MM tissues was significantly higher than that in BMN tissues $(P<0.01$; Fig. 5E), while the expression level of PTEN mRNA in MM tissues with metastasis was significantly lower than that in tissues without metastasis $(P<0.01$; Fig. 5F). Furthermore, the inverse correlation between miR-301a and PTEN mRNA expression in MM tissues could be testified by linear regression analysis (Spearman rank test $r=-0.388$; $P=0.026$ ) (Fig. 5G). Furthermore, we testified whether upregulation of PTEN can mimic the effects of miR-301a downregulation on phenotypes of MM cells. The A-375 cells were stably transfected with pMD/PTEN, and Western blot confirmed the upregulation of PTEN protein in A-375/PTEN, in comparison with A-375/control cells $(P<0.01$; Fig. $6 \mathrm{~A})$. Upregulation of PTEN significantly inhibited growth and colony formation, increased apoptosis and reduced the capacity of migration and invasion in MM cells (Fig. 6B-F). Also, it could be observed that upregulation of PTEN significantly enhanced chemosensitivity of MM cells by inceasing chemotherapy-induced apoptosis (Fig. 6G-H). From above data, it was concluded that PTEN was a direct and functional target of miR-301a in MM cells.

Akt and FAK signaling pathways are involved in miR-301a/PTEN-promoting malignant phenotypes in MM cells

It has been reported that activation of AKT and FAK signaling pathways plays critical roles in tumor growth and metastasis by regulating downstream signal molecules including Bcl-2, Bax, and MMP2/9. Meanwhile, PTEN can interact with AKT and FAK and inhibit their activity through their dephosphorylation [28]. We thus investigated whether Akt and FAK signaling pathways are involved in miR-301a/PTEN-promoting malignant phenotypes in MM cells. In this study, we showed that both miR-301a downregulation and PTEN upregulation in A-375 cells induced the decreased expression levels of phosphorylated AKT and FAK (p-Akt and p-FAK) and downstream proteins (Bcl-2, MMP-2 and MMP-9) and the increased expression level of Bax protein, but induce no changes in the total protein of Akt and FAK (Fig. 7A-B). Likewise, we also found that transfection of miR-301a/mimic induced the increased expression levels of p-Akt, p-FAK, Bcl-2, MMP-2 and MMP-9 proteins and the increased expression level of Bax protein, but induced no changes in the total protein of Akt and FAK in SK-EML-1 cells (Fig. 7C). More importantly, upregulation of PTEN reversed the changes of those proteins in SK-EML-1 cells induced by miR-301a/mimic (Fig. 7C). Together these findings suggest that miR-301a promotes MM progression via activation of Akt and FAK signaling pathways by down regulating PTEN.

\section{Discussion}

MM is characterized by aggressive invasion, early metastasis, and resistance to existing chemotherapeutic agents, and its development and progression are driven by complex genetic and epigenetic alterations [29]. Therefore, a better understanding of the molecular mechanism will contribute to finding novel approaches for both diagnosis and treatment to improve the formidable prognosis of advanced MM patients.

Recently, genome-wide studies have shown that the human genome is pervasively transcribed and produces many thousands of regulatory non-protein-coding RNAs (ncRNAs), which are well recognized as versatile and effective regulatory molecules in a wide range of 


\section{Cellular Physiology Cell Physiol Biochem 2016;40:230-244 \\ \begin{tabular}{l|l|l} 
and BOI: 10.1159/000452540 & $\begin{array}{l}\text { (c) 2016 The Author(s). Published by S. Karger AG, Basel } \\
\text { www.karger.com/cpb }\end{array}$
\end{tabular} \\ Cui et al.: MiR-301a: a Potential Therapeutic Target for Malignant Melanoma}

physiological and pathological processes, including tumorigenesis [30, 31]. Currently, ncRNAs include miRNAs, small interfering RNAs, PIWI-interacting RNAs and various classes of long ncRNAs. MiRNAs trigger mRNA degradation, stability or inhibition of translation by binding to specific target mRNA [32]. Accumulating evidence has suggested that dysregulation of miRNAs exerts an important role in MM progression by functioning as oncogenes or tumor suppressors [33]. Although many dysregulated miRNAs have been identified in MM, it is believed that there are still a large number of miRNAs uncovered and their unknown roles in MM progression need to be investigated. MiR-301a belongs to the miR-130 family, which is composed of miR-130b, miR-301a, and miR-301b. It has been reported that miR130 family regulates the hypoxia response signal through the P-body protein DDX6 [34]. Also, the microRNA-130/301 family is reported to control vasoconstriction in pulmonary hypertension [35]. Dou and his colleagues showed that miR-301a mediates the effect of IL-6 on the AKT/GSK pathway and hepatic glycogenesis by regulating PTEN expression [36]. Also, miR-301a is reported to promote colorectal cancer cell growth and invasion by directly targeting SOCS6 [37]. Meanwhile, Egawa and his colleagues showed that the miR130 family promotes cell migration and invasion in bladder cancer through FAK and Akt phosphorylation by regulating PTEN [38]. The current study focused on the functions of miR-301a and its molecular mechanisms in MM. Although Sand and his colleagues showed that miR-301a is upregulated in MM tissues, the correlations of miR-301a with metastasis and prognosis of MM patients are not investigated. Here, we showed that the expression level of miR-301a MM tissues with metastasis was higher than that in tissues without metastasis, suggesting that miR-301a may be a promoter in MM metastasis. Meanwhile, it was found that MM patients with high miR-301a expression showed poorer prognosis. However, whether the expression of miR-301 is an independent prognostic factor for MM patients still needs to be further analyzed in a larger case population. Next, we analyzed the effects of miR-301a expression on phenotypes of MM cells. Downregulation of miR-301a could significantly reduce the capacities of growth and colony formation and enhance apoptosis in MM cells, while upregulation of miR-301a induced the adverse effects in MM cells. These data suggest that miR-301a exerts growth-promoting effects in MM cells. As with other cancers, metastases occur when the MM is not caught in the early stages. Thus, understanding of the molecular mechanisms of MM metastasis is very meaningful. The correlations of dysregulated miRNAs with MM metastasis are also reported by other researchers. For example, miR-542-3p was reported to suppress invasion and metastasis by targeting the proto-oncogene serine/threonine protein kinase (PIM1) in melanoma [39]. Also, miR-382 was identified as a metastasis-suppressive microRNA in primary melanoma via regulation of actin regulators CTTN, RAC1, and ARPC2. Of course, MM metastasis is a complex process involving numerous changes of miRNAs [40]. In this study, we also showed that miR-301a promotes in vitro migration and invasion of MM cells. In future study, we will the effect of miR-301a on the in vivo metastasis of MM cells. In spite of highly effective new therapeutic strategies, chemotherapy still is an important treatment option for metastatic MM [41]. However, acquired chemoresistance is responsible for the therapeutic failure, thus there is an urgent need to delineate the underlying molecular mechanism of MM chemoresistance so as to identify novel therapeutic targets for this aggressive disease. Here, we showed that miR-301a downregulation could significantly increase the sensitivity of MM cells to CDDP or ADR by increasing chemotherapy-induced apoptosis. Likewise, miR-301a upregulation could reduce the sensitivity of MM cells to CDDP or ADR by decreasing chemotherapyinduced apoptosis. These data indicated that transfection of miR-301a/inhibitor might be a potential strategy for chemosensitization of MM.

It is well known that miRNAs exert their functions via target downregulation. Therefore, identification of gene targets is critical for functional characterization of miRNAs. Here, PTEN was identified as a putative target of miR-301a by three miRNA-target interactions online databases. PTEN is a phosphatase that removes phosphates primarily from lipids, and functions as a tumor suppressor gene that occupies a key position in regulating cell growth, apoptosis, metastasis, and chemo-radioresistance [42]. In malignant melanoma, 
epigenetic PTEN silencing without PTEN mutation has been reported [43]. Meanwhile, it was also reported that PTEN functions as a melanoma tumor suppressor by promoting host immune response [44]. Recently, the post-transcriptional regulation of PTEN by miR-301a was reported in Ewing's sarcoma and breast cancer [20, 27]. However, whether miR-301a functions in MM by targeting PTEN is unclear. The following experimental results of this study were summarised. First, luciferase reporter assays indicated that miR-301a could bind to the 3'-UTR of PTEN mRNA in MM cells. Second, transfection of miR-301a/mimic induced the decreased expression of PTEN mRNA and protein, while transfection of miR-301a/inhibitor induced the increased expression of PTEN mRNA and protein. Third, the expression of miR301a was negatively correlated with PTEN mRNA expression. Fourth, upregulation of PTEN could mimic the effects of miR-301a downregulation in MM cells. The above data indicated that PTEN was a direct and functional target of miR-301a in MM. PTEN is reported to down modulate signaling pathways that involve Akt and focal adhesion kinase (FAK) signaling pathways in tumor cells [45]. Here, we showed that both miR-301a downregulation and PTEN upregulation could reduce the expression of p-AKT and p-FAK proteins, which consequently led to downregulation of Bcl-2, MMP-2/9 and upregulation of Bax. Meanwhile, re-expression of miR-301a could induce adverse effects on those proteins in MM cells, and importantly, upregulation of PTEN could partially reverse the changes of those proteins induced by miR301a upregulation. Thus, activation of Akt and FAK signaling pathways might be involved in miR-301a/PTEN-promoting phenotypes in MM cells. The current study has several limits. First, the clinicopathological significance of miR-301a needs to be further analyzed. Second, the effects of miR-301a on the in vivo metastasis and chemosensitivity of MM cells remain to be testified in nude mouse xenograft model. Third, the other targets of miR-301a in MM need to be elucidated.

Taken together, this study indicated that upregulation of miR-301a correlated with metastasis and poor prognosis of MM patients. Functional assays showed that miR-301a promotes growth, migration, invasion and chemo resistance of MM cells via activation of Akt and FAK signals, at least partially by targeting PTEN. Therefore, miR-301a may be a potential molecular target for the treatment of human MMs.

\section{Disclosure Statement}

The authors declare that they have no competing interests.

\section{References}

1 Siegel R, Ma J, Zou Z, Jemal A: Cancer statistics, 2014. CA Cancer J Clin 2014;64:9-29.

2 Gray-Schopfer V, Wellbrock C, Marais R. Melanoma biology and new targeted therapy. Nature 2007;445:851-857.

3 Garbe C, Leiter U: Melanoma epidemiology and trends. Clin Dermatol 2009;27:3-9.

4 Ambros V: microRNAs: tiny regulators with great potential. Cell 2001;107:823-826.

5 Maul J, Baumjohann D: Emerging Roles for MicroRNAs in T Follicular Helper Cell Differentiation. Trends Immunol 2016;37:297-309.

6 Rottiers V, Näär AM: MicroRNAs in metabolism and metabolic disorders. Nat Rev Mol Cell Biol 2012;13:239-250.

7 Pileczki V, Cojocneanu-Petric R, Maralani M, Neagoe IB, Sandulescu R. MicroRNAs as regulators of apoptosis mechanisms in cancer. Clujul Med 2016;89:50-55.

8 Horak M, Novak J, Bienertova-Vasku J: Muscle-specific microRNAs in skeletal muscle development. Dev Biol 2016;410:1-13.

9 McManus MT: MicroRNAs and cancer. Semin Cancer Biol 2003;13:253-258.

10 Saldanha G, Elshaw S, Sachs P, Alharbi H, Shah P, Jothi A, Pringle JH: microRNA-10b is a prognostic biomarker for melanoma. Mod Pathol 2016;29:112-121. 


\section{Cellular Physiology Cell Physiol Biochem 2016;40:230-244 \begin{tabular}{l|l|l}
\hline DOI: 10.1159/000452540 & $\begin{array}{l}\text { C) 2016 The Author(s). Published by S. Karger AG, Basel } \\
\text { www.karger.com/cpb }\end{array}$
\end{tabular}}

Cui et al.: MiR-301a: a Potential Therapeutic Target for Malignant Melanoma

11 Lin N, Zhou Y, Lian X, Tu Y: Expression of microRNA-106b and its clinical significance in cutaneous melanoma. Genet Mol Res 2015;14:16379-16385.

12 Tian R, Liu T, Qiao L, Gao M, Li J: Decreased serum microRNA-206 level predicts unfavorable prognosis in patients with melanoma. Int J Clin Exp Pathol 2015;8:3097-3103.

13 Rang Z, Yang G, Wang YW, Cui F: miR-542-3p suppresses invasion and metastasis by targeting the proto-oncogene serine/threonine protein kinase, PIM1, in melanoma. Biochem Biophys Res Commun 2016;474:315-320.

14 Sun L, Bian G, Meng Z, Dang G, Shi D, Mi S: MiR-144 Inhibits Uveal Melanoma Cell Proliferation and Invasion by Regulating c-Met Expression. PLoS One 2015;10:e0124428.

15 Ren JW, Li ZJ, Tu C: MiR-135 post-transcriptionally regulates FOXO1 expression and promotes cell proliferation in human malignant melanoma cells. Int J Clin Exp Pathol 2015;8:6356-63566.

16 Sun L, Wang Q, Gao X, Shi D, Mi S, Han Q: MicroRNA-454 functions as an oncogene by regulating PTEN in uveal melanoma. FEBS Lett 2015;589:2791-2796.

17 Zhou P, Jiang W, Wu L, Chang R, Wu K, Wang Z: miR-301a is a candidate oncogene that targets the homeobox gene Gax in human hepatocellular carcinoma. Dig Dis Sci 2012;57:1171-1180.

18 Chen Z, Chen LY, Dai HY, Wang P, Gao S, Wang K: miR-301a promotes pancreatic cancer cell proliferation by directly inhibiting Bim expression. J Cell Biochem 2012;113:3229-3235.

19 Sand M, Skrygan M, Sand D, Georgas D, Gambichler T, Hahn SA, Altmeyer P, Bechara FG: Comparative microarray analysis of microRNA expression profiles in primary cutaneous malignant melanoma, cutaneous malignant melanoma metastases, and benign melanocytic nevi. Cell Tissue Res 2013;351:85-98.

20 Kawano M, Tanaka K, Itonaga I, Iwasaki T, Tsumura H: MicroRNA-301a promotes cell proliferation via PTEN targeting in Ewing's sarcoma cells. Int J Oncol 2016;48:1531-1540.

21 Bustin SA, Benes V, Garson JA, Hellemans J, Huggett J, Kubista M, Mueller R, Nolan T, Pfaffl MW, Shipley GL, Vandesompele J, Wittwer CT: The MIQE guidelines: minimum information for publication of quantitative real-time PCR experiments. Clin Chem 2009;55:611-622.

22 Chen D, Huang J, Zhang K, Pan B, Chen J, De W, Wang R, Chen L: MicroRNA-451 induces epithelialmesenchymal transition in docetaxel-resistant lung adenocarcinoma cells by targeting proto-oncogene c-Myc. Eur J Cancer 2014;50:3050-3067.

23 Zhang J, Zhang HD, Yao YF, Zhong SL, Zhao JH, Tang JH: $\beta$-Elemene Reverses Chemoresistance of Breast Cancer Cells by Reducing Resistance Transmission via Exosomes. Cell Physiol Biochem 2015;36:22742286.

24 Cui SY, Huang JY, Chen YT, Song HZ, Feng B, Huang GC, Wang R, Chen LB, De W: Let-7c governs the acquisition of chemo- or radioresistance and epithelial-to-mesenchymal transition phenotypes in docetaxel-resistant lung adenocarcinoma. Mol Cancer Res 2013;11:699-713.

25 Zhang J, Zhang Hd, Chen L, Sun DW, Mao Cf, Chen W, Wu JZ, Zhong SL, Zhao JH, Tang JH: $\beta$-elemene reverses chemoresistance of breast cancer via regulating MDR-related microRNA expression. Cell Physiol Biochem 2014;34:2027-2037.

26 Chen Q, Qin R, Fang Y, Li H: Berberine Sensitizes Human Ovarian Cancer Cells to Cisplatin Through miR-93/ PTEN/Akt Signaling Pathway. Cell Physiol Biochem 2015;36:956-965.

27 Ma F, Zhang J, Zhong L, Wang L, Liu Y, Wang Y, Peng L, Guo B: Upregulated microRNA-301a in breast cancer promotes tumor metastasis by targeting PTEN and activating Wnt/ $\beta$-catenin signaling. Gene 2014;535:191-197.

28 Xu W, Yang Z, Zhou SF, Lu N: Posttranslational regulation of phosphatase and tensin homolog (PTEN) and its functional impact on cancer behaviors. Drug Des Devel Ther 2014;8:1745-1751.

29 Tsao H, Atkins MB, Sober AJ: Management of cutaneous melanoma. N Engl J Med 2004;351:998-1012.

30 Sandhu GK, Milevskiy MJ, Wilson W, Shewan AM, Brown MA: Non-coding RNAs in Mammary Gland Development and Disease. Adv Exp Med Biol 2016;886:121-153.

31 Liz J, Esteller M: IncRNAs and microRNAs with a role in cancer development. Biochim Biophys Acta 2016;1859:169-176.

32 Ke XS, Liu CM, Liu DP, Liang CC: MicroRNAs: key participants in gene regulatory networks. Curr Opin Chem Biol 2003;7:516-523.

33 Sun V, Zhou WB, Majid S, Kashani-Sabet M, Dar AA: MicroRNA-mediated regulation of melanoma. Br J Dermatol 2014;171:234-241. 


\section{Cellular Physiology Cell Physiol Biochem 2016;40:230-244 \begin{tabular}{ll|l} 
DOI: 10.1159/000452540 & $\begin{array}{l}\text { O 2016 The Author(s). Published by S. Karger AG, Basel } \\
\text { munw karger.com/cpb }\end{array}$
\end{tabular} \\ Cui et al.: MiR-301a: a Potential Therapeutic Target for Malignant Melanoma}

34 Saito K, Kondo E, Matsushita M: MicroRNA 130 family regulates the hypoxia response signal through the P-body protein DDX6. Nucleic Acids Res 2011;39:6086-6099.

35 Bertero T, Cottrill K, Krauszman A, Lu Y, Annis S, Hale A, Bhat B, Waxman AB, Chau BN, Kuebler WM, Chan SY: The microRNA-130/301 family controls vasoconstriction in pulmonary hypertension. J Biol Chem 2015;290:2069-2085.

36 Dou L, Wang S, Sui X, Meng X, Shen T, Huang X, Guo J, Fang W, Man Y, Xi J, Li J: MiR-301a mediates the effect of IL-6 on the AKT/GSK pathway and hepatic glycogenesis by regulating PTEN expression. Cell Physiol Biochem 2015;35:1413-1424.

37 Fang Y, Sun B, Xiang J, Chen Z: MiR-301a promotes colorectal cancer cell growth and invasion by directly targeting SOCS6. Cell Physiol Biochem 2015;35:227-236.

38 Egawa H, Jingushi K, Hirono T, Ueda Y, Kitae K, Nakata W, Fujita K, Uemura M, Nonomura N, Tsujikawa K: The miR-130 family promotes cell migration and invasion in bladder cancer through FAK and Akt phosphorylation by regulating PTEN. Sci Rep 2016;6:20574.

39 Rang Z, Yang G, Wang YW, Cui F: miR-542-3p suppresses invasion and metastasis by targeting the proto-oncogene serine/threonine protein kinase, PIM1, in melanoma. Biochem Biophys Res Commun 2016;474:315-320.

40 Hanniford D, Segura MF, Zhong J, Philips E, Jirau-Serrano X, Darvishian F, Berman RS, Shapiro RL, Pavlick AC, Brown B, Osman I, Hernando E: Identification of metastasis-suppressive microRNAs in primary melanoma. J Natl Cancer Inst 2015;107(3).

41 Wilson MA, Schuchter LM: Chemotherapy for Melanoma. Cancer Treat Res 2016;167:209-229.

42 Milella M, Falcone I, Conciatori F, Cesta Incani U, Del Curatolo A, Inzerilli N, Nuzzo CM, Vaccaro V, Vari S, Cognetti F, Ciuffreda L: PTEN: Multiple Functions in Human Malignant Tumors. Front Oncol 2015;5:24.

43 Zhou XP, Gimm O, Hampel H, Niemann T, Walker MJ, Eng C: Epigenetic PTEN silencing in malignant melanomas without PTEN mutation. Am J Pathol 2000;157:1123-1128.

44 Dong Y, Richards JA, Gupta R, Aung PP, Emley A, Kluger Y, Dogra SK, Mahalingam M, Wajapeyee N: PTEN functions as a melanoma tumor suppressor by promoting host immune response. Oncogene 2014;33:4632-4642.

45 Yamada KM, Araki M: Tumor suppressor PTEN: modulator of cell signaling, growth, migration and apoptosis. J Cell Sci 2001;114:2375-2382. 